\title{
On almost periodicity of solutions of second-order differential equations involving reflection of the argument
}

\author{
Peiguang Wang ${ }^{1}$, Dhaou Lassoued ${ }^{2}$, Syed Abbas ${ }^{3}$, Akbar Zada ${ }^{4}$ and Tongxing Li $i^{5,6^{*}}$
}

\begin{tabular}{l}
\hline${ }^{\text {*Correspondence: }}$ \\
litongx2007@163.com \\
${ }^{5}$ LinDa Institute of Shandong \\
Provincial Key Laboratory of \\
Network Based Intelligent \\
Computing, Linyi University, Linyi, \\
P.R. China \\
${ }^{6}$ School of Information Science and \\
Engineering, Linyi University, Linyi, \\
P.R. China \\
Full list of author information is \\
available at the end of the article
\end{tabular}

available at the end of the article

\begin{abstract}
We study almost periodic solutions for a class of nonlinear second-order differential equations involving reflection of the argument. We establish existence results of almost periodic solutions as critical points by a variational approach. We also prove structure results on the set of strong almost periodic solutions, existence results of weak almost periodic solutions, and a density result on the almost periodic forcing term for which the equation possesses usual almost periodic solutions.
\end{abstract}

MSC: 34K14; 47H30; 58E30

Keywords: Almost periodic solution; Second-order differential equation; Reflection of the argument; Variational principle

\section{Introduction}

The study of existence, uniqueness, and stability of periodic and almost periodic solutions has become one of the most attractive topics in the qualitative theory of ordinary and functional differential equations for its significance in the physical sciences, mathematical biology, control theory, and other fields; see, for instance, $[3,8,11,19,20,28]$ and the references cited therein. Indeed, the almost periodic functions are closely connected with harmonic analysis, differential equations, and dynamical systems; cf. Corduneanu [12] and Fink [14]. These functions are basically generalizations of continuous periodic and quasi-periodic functions. Almost periodic functions are further generalized by many mathematicians in various ways; see Šarkovskii [26].

On the other hand, differential equations involving reflection of the argument have numerous applications in the study of stability of differential-difference equations. Such equations show very interesting properties by themselves, and so many authors have worked on this category of equations. Wiener and Aftabizadeh [29] initiated the analysis of boundary value problems involving reflection of the argument. Later on, Gupta [1517] considered boundary value problems for this class of equations. Aftabizadeh et al. [1] studied the existence of a unique bounded solution of the equation

$$
x^{\prime}(t)=f(t, x(t), x(-t)), \quad t \in \mathbb{R}
$$

(c) The Author(s) 2019. This article is distributed under the terms of the Creative Commons Attribution 4.0 International License (http://creativecommons.org/licenses/by/4.0/), which permits unrestricted use, distribution, and reproduction in any medium, provided you give appropriate credit to the original author(s) and the source, provide a link to the Creative Commons license, and indicate if changes were made. 
They proved that $t \mapsto x(t)$ is almost periodic by assuming the existence of bounded solutions. Further results were extended and improved by several authors; see, for instance, the papers by Hai [18], O’Regan [22], Piao [23, 24], Piao and Sun [25], and Zima [30]. In particular, Piao [23, 24] investigated the existence and uniqueness of periodic, almost periodic, and pseudo almost periodic solutions of the equations

$$
x^{\prime}(t)+a x(t)+b x(-t)=g(t), \quad b \neq 0, t \in \mathbb{R},
$$

and

$$
x^{\prime}(t)+a x(t)+b x(-t)=f(t, x(t), x(-t)), \quad b \neq 0, t \in \mathbb{R},
$$

whereas Piao and Sun [25] studied the existence and uniqueness of Besicovitch almost periodic solutions for a class of second-order differential equations involving reflection of the argument.

In the sequel, the linear space $\mathbb{R}^{n}$ is endowed with its standard inner product $x \cdot y:=$ $\sum_{k=1}^{n} x_{k} y_{k}$ and $|\cdot|$ denotes the associated Euclidean norm. For a function $f: \mathbb{R}^{n} \times \mathbb{R}^{n} \rightarrow \mathbb{R}$, $(X, Y) \mapsto f(X, Y)$, we consider the second-order differential equation with reflection of the argument

$$
u^{\prime \prime}(t)=D_{1} f(u(t), u(-t))+D_{2} f(u(-t), u(t))+e(t), \quad t \in \mathbb{R},
$$

where $D_{1}$ and $D_{2}$ denote the (partial) differential with respect to $X$ and $Y$, respectively, $e$ : $\mathbb{R} \rightarrow \mathbb{R}^{n}$ is an almost periodic forcing term. Equation (1.1) appears as an Euler-Lagrange equation.

By a strong almost periodic solution of equation (1.1) we mean a function $u: \mathbb{R} \rightarrow \mathbb{R}^{n}$ which is twice differentiable (in ordinary sense) such that $u, u^{\prime}$, and $u^{\prime \prime}$ are almost periodic in the sense of Bohr [9] and $u$ satisfies (1.1) for all $t \in \mathbb{R}$. This solution is also called $\mathcal{C}^{2}$ almost periodic in some earlier work.

A weak almost periodic solution of equation (1.1) is a function $u: \mathbb{R} \rightarrow \mathbb{R}^{n}$ which is almost periodic in the sense of Besicovitch [4] and possesses a first-order and a secondorder generalized derivatives such that $u$ satisfies (1.1) for all $t \in \mathbb{R}$ and the difference between the two members of equation (1.1) has a quadratic mean value equal to zero. It is natural that a strong almost periodic solution is also a weak almost periodic solution.

The variational method was used for the study of ordinary and functional differential equations; see, for instance, $[3,5-7]$ and the references cited therein. By using a variational method in the mean, we investigate almost periodic solutions for equation (1.1). The almost periodic solutions of (1.1) are characterized as critical points of functionals having the following form:

$$
u \mapsto \lim _{T \rightarrow \infty} \frac{1}{2 T} \int_{-T}^{T}\left(\frac{1}{2}\left|u^{\prime}(t)\right|^{2}+f(u(t), u(-t))+e(t) \cdot u(t)\right) d t
$$

on the Banach space of almost periodic functions.

This paper is organized as follows. Section 2 presents the considered notation for the various function spaces and auxiliary assumptions. In Sect. 3, we develop variational principles to study the almost periodic solutions of (1.1) and critical points of functionals defined on spaces of almost periodic functions. In Sect. 4, we establish some results about 
the structure of the set of strong almost periodic solutions of (1.1). Finally, in Sect. 5, we establish an existence result of weak almost periodic solutions of (1.1) by using the techniques in the spirit of the direct methods of calculus of variations, and a result on the density of the almost periodic forcing term for which (1.1) possesses a strong almost periodic solution.

\section{Notation and preliminaries}

First, we review some facts about Bohr almost periodic and Besicovitch almost periodic functions. For more details on almost periodic functions, we refer the reader to the monographs $[4,9,12,14,21]$.

Let $A P^{0}\left(\mathbb{R}^{n}\right)$ be the space of the almost periodic functions from $\mathbb{R}$ into $\mathbb{R}^{n}$ in the sense of Bohr [9], endowed with the norm

$$
\|u\|_{\infty}=\sup \{|u(t)|: t \in \mathbb{R}\} .
$$

It is easy to see that the space $A P^{0}\left(\mathbb{R}^{n}\right)$ is a Banach space [9] endowed with the above norm.

$\mathbb{N}$ is the set of all nonnegative integers, for $1 \leq k \in \mathbb{N}, A P^{k}\left(\mathbb{R}^{n}\right)$ stands for the space of functions $u \in \mathcal{C}^{k}\left(\mathbb{R}, \mathbb{R}^{n}\right) \cap A P^{0}\left(\mathbb{R}^{n}\right)$ such that $u^{(j)}:=\frac{d u^{j} u}{d t^{j}} \in A P^{0}\left(\mathbb{R}^{n}\right)$ for all $j=1, \ldots, k$. It is a Banach space endowed with the norm

$$
\|u\|_{\mathcal{C}^{k}}=\|u\|_{\infty}+\sum_{j=1}^{k}\left\|u^{(j)}\right\|_{\infty} .
$$

Every almost periodic function $u$ possesses a mean time

$$
\mathcal{M}\{u\}=\mathcal{M}\{u(t)\}_{t}:=\lim _{T \rightarrow \infty} \frac{1}{2 T} \int_{-T}^{T} u(t) d t .
$$

For $\lambda \in \mathbb{R}, a(u, \lambda):=\mathcal{M}\left\{u(t) e^{-i \lambda t}\right\}_{t}$ is the Fourier-Bohr coefficient of $u$ associated to $\lambda$. We denote by $\Lambda(u):=\{\lambda \in \mathbb{R}: a(u, \lambda) \neq 0\}$ the set of exponents of $u$. We use the notation $\bmod (u)$ for the module of $u$ which is the additive group generated by $\Lambda(u)$.

Remark 2.1 For $p \in[1, \infty), B^{p}\left(\mathbb{R}^{n}\right)$ is the completion of $A P^{0}\left(\mathbb{R}^{n}\right)$ in $L_{\text {loc }}^{p}\left(\mathbb{R}, \mathbb{R}^{n}\right)$ (Lebesgue space) with respect to the norm

$$
\|u\|_{p}:=\mathcal{M}\left\{|u|^{p}\right\}^{\frac{1}{p}}
$$

Remark 2.2 For $p=2, B^{2}\left(\mathbb{R}^{n}\right)$ is a Hilbert space and its norm $\|\cdot\|_{2}$ is associated to the inner product $(u \mid v):=\mathcal{M}\{u \cdot v\}$. The elements of these spaces $B^{p}\left(\mathbb{R}^{n}\right)$ are called Besicovitch almost periodic functions, cf. [4].

Now, we recall the definitions of some spaces, like Sobolev space, special to the almost periodicity introduced by Blot [7]. Following Vo-Khac [27], the generalized derivative of $u \in B^{2}\left(\mathbb{R}^{n}\right)$ (when it exists) is $\nabla u \in B^{2}\left(\mathbb{R}^{n}\right)$ such that

$$
\lim _{\tau \rightarrow 0} \mathcal{M}\left\{\left|\nabla u(t)-\frac{u(t+\tau)-u(t)}{\tau}\right|^{2}\right\}_{t}=0 .
$$


The space $B^{1,2}\left(\mathbb{R}^{n}\right)$ is the collection of all functions $u \in B^{2}\left(\mathbb{R}^{n}\right)$ such that $\nabla u$ exists in $B^{2}\left(\mathbb{R}^{n}\right)$, and the space $B^{2,2}\left(\mathbb{R}^{n}\right)$ is the space of $u \in B^{1,2}\left(\mathbb{R}^{n}\right)$ such that $\nabla^{2} u=\nabla(\nabla u)$ exists in $B^{2}\left(\mathbb{R}^{n}\right)$. It is easy to verify that the above-mentioned spaces are Hilbert spaces with the respective norms

$$
\|u\|_{1,2}:=\left(\|u\|_{2}^{2}+\|\nabla u\|_{2}^{2}\right)^{\frac{1}{2}} \quad \text { and } \quad\|u\|_{2,2}:=\left(\|u\|_{2}^{2}+\|\nabla u\|_{2}^{2}+\left\|\nabla^{2} u\right\|_{2}^{2}\right)^{\frac{1}{2}} .
$$

For the function $f: \mathbb{R}^{n} \times \mathbb{R}^{n} \longrightarrow \mathbb{R},(x, y) \mapsto f(x, y)$ of equation (1.1), we give the following hypotheses:

$\left(\mathrm{H}_{1}\right) f \in \mathcal{C}^{1}\left(\mathbb{R}^{n} \times \mathbb{R}^{n}, \mathbb{R}\right)$;

$\left(\mathrm{H}_{2}\right)|D f(X)-D f(Y)| \leq a \cdot|X-Y|$ for some constant $a>0$ and for all $X, Y \in \mathbb{R}^{n} \times \mathbb{R}^{n}$;

$\left(\mathrm{H}_{3}\right) f$ is a convex function on $\mathbb{R}^{n} \times \mathbb{R}^{n}$;

$\left(\mathrm{H}_{4}\right) f(x, y) \geq c|\zeta|^{2}+d$ for two numbers $c>0$ and $d \in \mathbb{R}$ and for all $(x, y) \in \mathbb{R}^{n} \times \mathbb{R}^{n}$, where $\zeta=x$ or $y$.

\section{Variational principles}

We begin this section by establishing two lemmas which contain general properties of almost periodic functions.

Lemma 3.1 If $u \in A P^{0}\left(\mathbb{R}^{n}\right)$, then $[t \mapsto u(-t)] \in A P^{0}\left(\mathbb{R}^{n}\right)$. Furthermore, if $\tau$ is an $\epsilon$ translation of $u(t)$, then $\tau$ is also an $\epsilon$-translation number of $u(-t)$ and $\bmod (u(t))=$ $\bmod (u(-t))$.

Proof The proof can be completed by using Bohr's definition [9, p. 32].

Lemma 3.2 If $u \in B^{p}\left(\mathbb{R}^{n}\right)$, then the following assertions hold.

(1) $\mathcal{M}\{u(t)\}_{t}=\mathcal{M}\{u(-t)\}_{t}$.

(2) $[t \mapsto u(-t)] \in B^{p}\left(\mathbb{R}^{n}\right)$.

Proof The relation

$$
\mathcal{M}\{u(-t)\}_{t}=\lim _{T \rightarrow \infty} \frac{1}{2 T} \int_{-T}^{T} u(-t) d t=\lim _{T \rightarrow \infty} \frac{1}{2 T} \int_{T}^{-T}-u(s) d s=\mathcal{M}\{u(t)\}_{t}
$$

gives assertion (1). For assertion (2), note that if $\left(u_{m}\right)_{m}$ is a sequence in $A P^{0}\left(\mathbb{R}^{n}\right)$ such that $\lim _{m \rightarrow \infty}\left\|u-u_{m}\right\|_{p}=0$, then using Lemma 3.1 and the facts that $\left(u_{m}(-t)\right)_{m}$ is a sequence in $A P^{0}\left(\mathbb{R}^{n}\right)$ and $\left\|u-u_{m}\right\|_{p}=\left\|u(-t)-u_{m}(-t)\right\|_{p}$, we obtain

$$
\lim _{m \rightarrow \infty}\left\|u(-t)-u_{m}(-t)\right\|_{p}=0
$$

which implies that $[t \mapsto u(-t)] \in B^{p}\left(\mathbb{R}^{n}\right)$. The proof is complete.

Lemma 3.3 Under condition $\left(H_{1}\right)$, the functional $J_{0}: A P^{1}\left(\mathbb{R}^{n}\right) \rightarrow \mathbb{R}$ defined by

$$
J_{0}(u):=\mathcal{M}\left\{\frac{1}{2}\left|u^{\prime}(t)\right|^{2}+f(u(t), u(-t))+e(t) \cdot u(t)\right\}_{t}
$$


is of class $\mathcal{C}^{1}$, and for all $u, v \in A P^{0}\left(\mathbb{R}^{n}\right)$,

$$
\begin{aligned}
D_{0}(u) \cdot v= & \mathcal{M}\left\{u^{\prime}(t) \cdot v^{\prime}(t)+D_{1} f(u(t), u(-t)) \cdot v(t)\right. \\
& \left.+D_{2} f(u(t), u(-t)) \cdot v(-t)+e(t) \cdot v(t)\right\}_{t} .
\end{aligned}
$$

Proof We consider the operator $Q_{0}: A P^{1}\left(\mathbb{R}^{n}\right) \rightarrow \mathbb{R}$ defined by $Q_{0}(u):=\mathcal{M}\left\{\frac{1}{2}\left|u^{\prime}\right|^{2}\right\}$. The mapping $q: \mathbb{R}^{n} \rightarrow \mathbb{R}, q(x)=\frac{1}{2}|x|^{2}$, is of class $\mathcal{C}^{1}$, so the Nemytskii operator $\mathcal{N}_{q}^{0}: A P^{0}\left(\mathbb{R}^{n}\right) \rightarrow$ $A P^{0}(\mathbb{R}), \mathcal{N}_{q}^{0}(\phi):=\left[t \mapsto \frac{1}{2}|\phi(t)|^{2}\right]$, is of class $\mathcal{C}^{1}$, cf. [5]. The operator $\frac{d}{d t}: A P^{1}\left(\mathbb{R}^{n}\right) \rightarrow$ $A P^{0}\left(\mathbb{R}^{n}\right)$ defined by $\frac{d}{d t}(u):=u^{\prime}$ is linear continuous, therefore, it is of class $\mathcal{C}^{1}$. The functional $\mathcal{M}^{0}: A P^{0}(\mathbb{R}) \rightarrow \mathbb{R}$ defined by $\mathcal{M}^{0}(\phi):=\mathcal{M}_{t}^{0}\{\phi(t)\}$ is linear continuous, and hence it is of class $\mathcal{C}^{1}$.

Since $Q_{0}=\mathcal{M}^{0} \circ \mathcal{N}_{q}^{0} \circ \frac{d}{d t}, Q_{0}$ is of class $\mathcal{C}^{1}$ as composition of $\mathcal{C}^{1}$-mappings. Hence, by the chain rule, we have $D Q_{0}(u) v=\mathcal{M}\left\{u^{\prime} \cdot v^{\prime}\right\}$.

Furthermore, the operator $\Theta_{0}: A P^{1}\left(\mathbb{R}^{n}\right) \rightarrow \mathbb{R}$ defined by $\Theta_{0}(u):=\mathcal{M}\{e \cdot u\}$ is linear continuous, so it is of class $\mathcal{C}^{1}$ and its differential is given by $D \Theta_{0}(u) v=\mathcal{M}\{e \cdot v\}$.

We consider the operator $\Phi_{0}: A P^{1}\left(\mathbb{R}^{n}\right) \rightarrow \mathbb{R}$ defined by $\Phi_{0}(u):=\mathcal{M}\{f(u(t), u(-t))\}_{t}$. It is not difficult to observe that the operator $L_{0}: A P^{0}\left(\mathbb{R}^{n}\right) \rightarrow A P^{0}\left(\mathbb{R}^{n}\right) \times A P^{0}\left(\mathbb{R}^{n}\right)$ defined by $L_{0}(u)(t):=(u(t), u(-t))$ is linear. Both components of $L_{0}$ are continuous and hence $L_{0}$ is continuous. Therefore, $L_{0}$ is of class $\mathcal{C}^{1}$ and $D L_{0}(u) v=L_{0}(v)$ for all $u, v \in A P^{0}\left(\mathbb{R}^{n}\right)$.

Now, under assumption $\left(\mathrm{H}_{1}\right)$, the Nemytskii operator $\mathcal{N}_{f}^{0}: A P^{0}\left(\mathbb{R}^{n} \times \mathbb{R}^{n}\right) \rightarrow A P^{0}(\mathbb{R})$ defined by $\mathcal{N}_{f}^{0}(U)(t):=f(U(t))$ is of class $\mathcal{C}^{1}$ (see [6] for details). Moreover, for all $U, V \in$ $\left(A P^{0}\left(\mathbb{R}^{n}\right)\right)^{2}, D \mathcal{N}_{f}^{0}(U) \cdot V=D f(U) \cdot V$.

Note that the linear operator $\mathcal{M}_{0}: A P^{0}(\mathbb{R}) \rightarrow \mathbb{R}$ defined by $\mathcal{M}_{0}(u):=\mathcal{M}\{u(t)\}_{t}$ is continuous. It is of class $\mathcal{C}^{1}$ and thus $D \mathcal{M}_{0}(\phi) \psi=\mathcal{M}(\psi)$ for all $\phi, \psi \in A P^{0}(\mathbb{R})$. Further, the linear operator $i n_{0}: A P^{1}\left(\mathbb{R}^{n}\right) \rightarrow A P^{0}\left(\mathbb{R}^{n}\right), i n_{0}(u):=u$ is continuous, and consequently it is of class $\mathcal{C}^{1}$ and so $\operatorname{Din}_{0}(u) v=i n_{0}(v)$. Since $\Phi_{0}=\mathcal{M}_{0} \circ \mathcal{N}_{f}^{0} \circ L_{0} \circ i n_{0}, \Phi_{0}$ is of class $\mathcal{C}^{1}$ as a composition of $\mathcal{C}^{1}$ operators. Using the chain rule, for all $u, v \in A P^{1}\left(\mathbb{R}^{n}\right)$,

$$
\left(D \Phi_{0}(u) \cdot v\right)(t)=\mathcal{M}\left\{D_{1} f(u(t), u(-t)) \cdot v(t)+D_{2} f(u(t), u(-t)) \cdot v(-t)\right\}_{t} .
$$

By virtue of $J_{0}=Q_{0}+\Phi_{0}+\Theta_{0}, J_{0}$ is of class $\mathcal{C}^{1}$ as a sum of three $\mathcal{C}^{1}$ functionals. Therefore, for all $u, v \in A P^{1}\left(\mathbb{R}^{n}\right)$, we have (3.1). This completes the proof.

Lemma 3.4 Assume that assumptions $\left(\mathrm{H}_{1}\right)$ and $\left(\mathrm{H}_{2}\right)$ are satisfied. Then the Nemytskii operator $\mathcal{N}_{f}^{1}: B^{2}\left(\mathbb{R}^{n} \times \mathbb{R}^{n}\right) \rightarrow B^{1}(\mathbb{R})$ defined by $\mathcal{N}_{f}^{1}(U)(t):=f(U(t))$ is well defined and is of class $\mathcal{C}^{1}$, and $D \mathcal{N}_{f}^{1}(U) \cdot V=D f(U) \cdot V$ for all $U, V \in B^{2}\left(\mathbb{R}^{n} \times \mathbb{R}^{n}\right)$.

Proof It suffices to remark that if $\left(\mathrm{H}_{1}\right)$ and $\left(\mathrm{H}_{2}\right)$ hold, then, for all $X \in \mathbb{R}^{n}$,

$$
|D f(X)| \leq|D f(X)-D f(0)|+|D f(0)| \leq a|X|+|D f(0)| .
$$

Using the mean value theorem (see [2, p. 144]), we deduce that, for all $X \in \mathbb{R}^{n}$,

$$
\begin{aligned}
|f(X)| & \leq|f(X)-f(Y)|+|f(0)| \\
& \leq \sup _{\xi \in] 0, x[}|D f(\xi)||x|+|D f(0)|
\end{aligned}
$$




$$
\begin{aligned}
& \leq(a|X|+|D f(0)|)|X|+|D f(0)| \\
& \leq a|X|^{2}+|D f(0)||X|+|D f(0)| \\
& \leq a|X|^{2}+\frac{1}{2}|D f(0)|^{2}+\frac{1}{2}|X|^{2}+|D f(0)| \\
& =\left(a+\frac{1}{2}\right)|X|^{2}+\left(\frac{1}{2}|D f(0)|^{2}+|D f(0)|\right) .
\end{aligned}
$$

Now, arguing as in [7, Theorem 2], we obtain the result. The proof is complete.

Proposition 3.5 Under condition $\left(\mathrm{H}_{1}\right)$, the following assertions are equivalent.

(1) $u$ is a critical point of $J_{0}$ on $A P^{1}\left(\mathbb{R}^{n}\right)$.

(2) $u$ is a strong almost periodic solution of (1.1).

Proof Define

$$
q(t):=D_{1} f(u(t), u(-t))+D_{2} f(u(-t), u(t))+e(t) .
$$

We know that $q \in A P^{0}\left(\mathbb{R}^{n}\right)$ for $u \in A P^{0}\left(\mathbb{R}^{n}\right)$. Let us first assume assertion (1). Since the mean value is invariant by reflection of the argument, we have

$$
\mathcal{M}\left\{D_{2} f(u(t), u(-t)) \cdot v(-t)\right\}_{t}=\mathcal{M}\left\{D_{2} f(u(-t), u(t)) \cdot v(t)\right\}_{t} .
$$

Hence, by Lemma 3.3, for all $v \in A P^{1}\left(\mathbb{R}^{n}\right)$, we get $0=\mathcal{M}\left\{u^{\prime} \cdot v^{\prime}+q \cdot v\right\}$. Finally, by using the same reasoning as in the proof of [5, Theorem 1], we obtain $u \in A P^{2}\left(\mathbb{R}^{n}\right)$ and $u^{\prime \prime}=q$, which is exactly (1.1).

Conversely, if $u$ is a strong almost periodic solution of (1.1), then we have $u^{\prime \prime}=q$. Hence, for all $v \in A P^{1}\left(\mathbb{R}^{n}\right)$, we obtain

$$
D J_{0}(u) \cdot v=\mathcal{M}\left\{u^{\prime} \cdot v^{\prime}+q \cdot v\right\}=\mathcal{M}\left\{\frac{d}{d t}\left(u^{\prime} \cdot v\right)\right\}=0 .
$$

This completes the proof.

Lemma 3.6 If conditions $\left(\mathrm{H}_{1}\right)$ and $\left(\mathrm{H}_{2}\right)$ are fulfilled, then the functional $J_{1}: B^{1,2}\left(\mathbb{R}^{n}\right) \rightarrow \mathbb{R}$ defined by

$$
J_{1}(u):=\mathcal{M}\left\{\frac{1}{2}|\nabla u(t)|^{2}+f(u(t), u(-t))+e(t) \cdot u(t)\right\}_{t}
$$

is of class $\mathcal{C}^{1}$. Moreover, for all $u, v \in B^{1,2}\left(\mathbb{R}^{n}\right)$,

$$
\begin{aligned}
D J_{1}(u) \cdot v= & \mathcal{M}\left\{\nabla u(t) \cdot \nabla v(t)+D_{1} f(u(t), u(-t)) \cdot v(t)\right. \\
& \left.+D_{2} f(u(t), u(-t)) \cdot v(-t)+e(t) \cdot v(t)\right\}_{t} .
\end{aligned}
$$

Proof We consider the operator $Q_{1}: B^{1,2}\left(\mathbb{R}^{n}\right) \rightarrow \mathbb{R}$ defined by $Q_{1}(u):=\mathcal{M}\left\{\frac{1}{2}|\nabla u|^{2}\right\}$. The mapping $q: \mathbb{R}^{n} \rightarrow \mathbb{R}, q(x)=\frac{1}{2}|x|^{2}$, is of class $\mathcal{C}^{1}$. Since $D q(x)=x$ satisfies conditions of 
[13, Theorem 2.6], the Nemytskii operator $\mathcal{N}_{q}: B^{2}\left(\mathbb{R}^{n}\right) \rightarrow B^{1}(\mathbb{R})$ defined by $\mathcal{N}_{q}(v):=[t \mapsto$ $\left.\frac{1}{2}|v(t)|^{2}\right]$ is of class $\mathcal{C}^{1}$ and $D \mathcal{N}_{q}(v) \cdot h=[t \mapsto v(t) \cdot h(t)]$ for all $v, h \in B^{2}\left(\mathbb{R}^{n}\right)$.

Since the derivation operator $\nabla: B^{1,2}\left(\mathbb{R}^{n}\right) \rightarrow B^{2}(\mathbb{R})$ and the operator $\mathcal{M}: B^{1}(\mathbb{R}) \rightarrow \mathbb{R}$ are linear continuous, $\nabla$ and $\mathcal{M}$ are of class $\mathcal{C}^{1}$. Therefore, $Q_{1}=\mathcal{M} \circ \mathcal{N}_{q} \circ \nabla$ is of class $\mathcal{C}^{1}$ as a composition of $\mathcal{C}^{1}$-mappings. Moreover, using the chain rule, we have $D Q_{1}(u) \cdot v=$ $\mathcal{M}\{\nabla u \cdot \nabla v\}$ for all $u, v \in B^{1,2}\left(\mathbb{R}^{n}\right)$.

Now, the operator $\Theta_{1}: B^{1,2}\left(\mathbb{R}^{n}\right) \rightarrow \mathbb{R}$ defined by $\Theta_{1}(u):=\mathcal{M}\{e \cdot u\}$ is linear continuous, and thus it is of class $\mathcal{C}^{1}$ and its differential is given by $D \Theta_{1}(u) v=\mathcal{M}\{e \cdot v\}$.

Let us consider the operator $\Phi_{1}: B^{1,2}\left(\mathbb{R}^{n}\right) \rightarrow \mathbb{R}$ defined by $\Phi_{1}(u):=\mathcal{M}\{f(u(t), u(-t))\}_{t}$. Note that the linear operator $L_{1}: B^{2}\left(\mathbb{R}^{n}\right) \rightarrow B^{2}\left(\mathbb{R}^{n}\right) \times B^{2}\left(\mathbb{R}^{n}\right)$ defined by $L_{1}(u)(t):=$ $(u(t), u(-t))$ is continuous and so it is of class $\mathcal{C}^{1}$. Moreover, for all $u, v \in B^{2}\left(\mathbb{R}^{n}\right)$, we have $D L_{1}(u) v=L_{1}(v)$.

Under assumptions $\left(\mathrm{H}_{1}\right)$ and $\left(\mathrm{H}_{2}\right)$, by virtue of Lemma 3.4, the Nemytskii operator $\mathcal{N}_{f}^{1}: B^{2}\left(\mathbb{R}^{n} \times \mathbb{R}^{n}\right) \rightarrow B^{1}(\mathbb{R})$ defined by $\mathcal{N}_{f}^{1}(U)(t):=f(U(t))$ is of class $\mathcal{C}^{1}$ and for all $U, V \in B^{2}\left(\mathbb{R}^{n} \times \mathbb{R}^{n}\right), D \mathcal{N}_{f}^{1}(U) \cdot V=D f(U) \cdot V$.

The continuous linear operator $\mathcal{M}_{1}: B^{1}(\mathbb{R}) \rightarrow \mathbb{R}$ defined by $\mathcal{M}_{1}(u):=\mathcal{M}\{u(t)\}_{t}$ is of class $\mathcal{C}^{1}$ and for all $\phi, \psi \in B^{1}(\mathbb{R}), D \mathcal{M}_{1}(\phi) \psi=\mathcal{M}(\psi)$. Besides, the linear operator $i n_{1}$ : $B^{1,2}\left(\mathbb{R}^{n}\right) \rightarrow B^{2}\left(\mathbb{R}^{n}\right), i n_{1}(u)=u$ is of class $\mathcal{C}^{1}$ and $\operatorname{Din}_{1}(u) v=i n_{1}(v)$.

Since $\Phi_{1}=\mathcal{M}_{1} \circ \mathcal{N}_{f}^{1} \circ L_{1} \circ i n_{1}, \Phi_{1}$ is of class $\mathcal{C}^{1}$ as it is composition of $\mathcal{C}^{1}$ operators. Hence, by the chain rule, for all $u, v \in B^{1,2}\left(\mathbb{R}^{n}\right)$,

$$
\left(D \Phi_{1}(u) \cdot v\right)(t)=\mathcal{M}\left\{D_{1} f(u(t), u(-t)) \cdot v(t)+D_{2} f(u(t), u(-t)) \cdot v(-t)\right\}_{t} .
$$

By virtue of $J_{1}=Q_{1}+\Phi_{1}+\Theta_{1}, J_{1}$ is of class $\mathcal{C}^{1}$ as a sum of three $\mathcal{C}^{1}$ functionals. Thus, for all $u, v \in B^{1,2}\left(\mathbb{R}^{n}\right)$, we obtain (3.2). The proof is complete.

Proposition 3.7 Under conditions $\left(\mathrm{H}_{1}\right)$ and $\left(\mathrm{H}_{2}\right)$, the following assertions are equivalent.

(1) $u$ is a critical point of $J_{1}$ on $B^{1,2}\left(\mathbb{R}^{n}\right)$.

(2) $u$ is a weak almost periodic solution of (1.1).

Proof Set

$$
p(t):=D_{1} f(u(t), u(-t))+D_{2} f(u(-t), u(t))+e(t) .
$$

It is well known that $p \in B^{2}\left(\mathbb{R}^{n}\right)$ if $u \in B^{2}\left(\mathbb{R}^{n}\right)$. Now if we assume that $u \in B^{1,2}\left(\mathbb{R}^{n}\right)$ is a critical point of $J_{1}$, then the condition $D J_{1}(u)=0$ can be written as $\mathcal{M}\{\nabla u \cdot \nabla v\}=-\mathcal{M}\{p$. $v$ \} for all $v \in B^{1,2}\left(\mathbb{R}^{n}\right)$. Hence, using [7, Proposition 10], the last condition implies that $\nabla u \in B^{1,2}\left(\mathbb{R}^{n}\right)$, i.e., $u \in B^{2,2}\left(\mathbb{R}^{n}\right)$ and $\nabla^{2} u=p$, which exactly means that $u$ is a weak almost periodic solution of (1.1).

Conversely, assume that the assertion (2) is true. Then $\nabla u \in B^{1,2}\left(\mathbb{R}^{n}\right)$. Using the fact that $\mathcal{M}\{\nabla w\}=0$ for all $w \in B^{1,2}\left(\mathbb{R}^{n}\right)$ (see [7, Proposition 3] for details) and [7, Proposition 9], we have, for all $h \in A P^{1}\left(\mathbb{R}^{n}\right)$,

$$
\begin{aligned}
0 & =\mathcal{M}\{\nabla(\nabla u \cdot h)\} \\
& =\mathcal{M}\left\{\nabla^{2} u \cdot h\right\}+\mathcal{M}\left\{\nabla u \cdot h^{\prime}\right\}
\end{aligned}
$$




$$
\begin{aligned}
& =\mathcal{M}\{p \cdot h\}+\mathcal{M}\left\{\nabla u \cdot h^{\prime}\right\} \\
& =D J_{1}(u) \cdot h .
\end{aligned}
$$

Since $A P^{1}\left(\mathbb{R}^{n}\right)$ is dense in $B^{1,2}\left(\mathbb{R}^{n}\right)$, we have $D J_{1}(u) \cdot h=0$ for all $h \in B^{1,2}\left(\mathbb{R}^{n}\right)$. Therefore, $D J_{1}(u)=0$, which proves our claim. This completes the proof.

\section{Structure results on $A P^{0}\left(\mathbb{R}^{n}\right)$}

In this section, we give some structure results on the set of strong almost periodic solutions of equation (1.1). The main tool is the variational structure of the problem.

Theorem 4.1 Under assumptions $\left(\mathrm{H}_{1}\right)$ and $\left(\mathrm{H}_{3}\right)$, the following assertions hold.

(1) The set of the strong almost periodic solutions of (1.1) is a convex closed subset of $A P^{1}\left(\mathbb{R}^{n}\right)$.

(2) If $u_{1}$ is a $T_{1}$ periodic solution of (1.1), $u_{2}$ is a $T_{2}$ periodic solution of (1.1), and $T_{1} / T_{2}$ is not rational, then $(1-\theta) u_{1}+\theta u_{2}$ is a strong almost periodic but nonperiodic solution of (1.1) for all $\theta \in(0,1)$.

Proof Since $f$ is convex and is of class $\mathcal{C}^{1}$, the operator $J_{0}$ is convex and is of class $\mathcal{C}^{1}$ on $A P^{1}\left(\mathbb{R}^{n}\right)$. Therefore,

$$
\left\{u \in A P^{1}\left(\mathbb{R}^{n}\right): J_{0}(u)=\inf J_{0}\left(A P^{1}\left(\mathbb{R}^{n}\right)\right)\right\}=\left\{u \in A P^{1}\left(\mathbb{R}^{n}\right): D J_{0}(u)=0\right\}
$$

is closed and convex, and hence assertion (1) becomes a consequence of Proposition 3.5. The assertion (2) is a straightforward consequence of (1). The proof is complete.

Theorem 4.2 Under assumptions $\left(\mathrm{H}_{1}\right)$ and $\left(\mathrm{H}_{3}\right)$, if $e=0$, then the following assertions hold.

(1) If $u$ is a strong almost periodic solution of $(1.1)$ and $T \in(0, \infty)$ satisfies $a(u, 2 \pi / T) \neq 0$, then there exists a nonconstant $T$ periodic solution of (1.1).

(2) If $u$ is a strong almost periodic solution of (1.1), then $\mathcal{M}\{u\}$ is a constant solution of (1.1).

Proof Define $C_{T, v}(u)(t):=\frac{1}{v} \sum_{k=0}^{v-1} u(t+k T)$ for all $v \in \mathbb{N}^{*}$, where $u$ is a strong almost periodic solution of (1.1). According to the Besicovitch theorem [4, p. 144], there exists a $T$ periodic continuous function denoted by $u_{T}$ such that

$$
\lim _{\nu \rightarrow \infty}\left\|C_{T, v}(u)-u_{T}\right\|_{\infty}=0 .
$$

Thus we can easily verify that

$$
\lim _{v \rightarrow \infty}\left\|C_{T, v}(u)-u_{T}\right\|_{\mathcal{C}^{1}}=0
$$

Since $e=0, t \mapsto u(t+k T)$ is a strong almost periodic solution. Furthermore, since $C_{T, v}(u)$ is a convex combination of strong almost periodic solutions of (1.1), $C_{T, v}(u)$ is also a strong almost periodic solution of (1.1), and hence $u_{T}$ is also strong almost periodic by using the closedness of the set of strong almost periodic solutions. Thus $u_{T}$ is a $T$ periodic solution of (1.1). Now, using a straightforward calculation, we can easily observe that 
$a\left(C_{T, v}(u), \frac{2 \pi}{T}\right)=a\left(u, \frac{2 \pi}{T}\right)$ and consequently $a\left(u_{T}, \frac{2 \pi}{T}\right)=a\left(u, \frac{2 \pi}{T}\right) \neq 0$, then $u_{T}$ is not constant which proves assertion (1).

To prove assertion (2), it suffices to choose $T \in(0, \infty)$ such that $\frac{2 \pi}{T}(\mathbb{Z}-\{0\}) \cap \Lambda(u)=\emptyset$. So all the Fourier-Bohr coefficients of $u_{T}$ are zero except (perhaps) the mean value of $u_{T}$ which is equal to $\mathcal{M}\{u\}$. This completes the proof.

\section{Existence results}

In this section, we study the weak almost periodic solutions of equation (1.1). In the previous section, we use a variational viewpoint but here the Hilbert structure of $B^{2}\left(\mathbb{R}^{n}\right)$ permits us to obtain an existence theorem by using direct methods of calculus of variations. Finally, in Theorem 5.2, we give a result of density of the almost periodic forcing term for which equation (1.1) possesses usual almost periodic solutions.

Theorem 5.1 Under assumptions $\left(\mathrm{H}_{1}\right)-\left(\mathrm{H}_{4}\right)$, for each $e \in B^{2}\left(\mathbb{R}^{n}\right)$, there exists a $u \in$ $B^{2,2}\left(\mathbb{R}^{n}\right)$ which is a weak almost periodic solution of (1.1). Moreover, the set of the weak almost periodic solutions of (1.1) is a convex set.

Proof Using Lemma 3.6, under assumptions $\left(\mathrm{H}_{1}\right)$ and $\left(\mathrm{H}_{2}\right)$, the functional $J_{1}$ is of class $\mathcal{C}^{1}$. It follows from $\left(\mathrm{H}_{3}\right)$ that $J_{1}$ is a convex functional. Since the mean value is invariant by reflection, assumption $\left(\mathrm{H}_{4}\right)$ implies that, for all $u \in B^{1,2}\left(\mathbb{R}^{n}\right)$,

$$
J_{1}(u) \geq \frac{1}{2}\|u\|_{1,2}^{2}+c\|u\|_{2}^{2}-\|u\|_{2}\|e\|_{2} \geq \alpha\|u\|_{1,2}^{2}-\|u\|_{2}\|e\|_{2}
$$

where $\alpha:=\min \left\{\frac{1}{2}, c\right\}$. Consequently, $J_{1}$ is coercive on $B^{1,2}\left(\mathbb{R}^{n}\right)$, i.e., $J_{1}(u) \rightarrow \infty$ as $\|u\|_{1,2} \rightarrow$ $\infty$, and so (see $\left[10\right.$, p. 46]) there exists a $u \in B^{1,2}\left(\mathbb{R}^{n}\right)$ such that $J_{1}(u)=\inf J_{1}\left(B^{1,2}\left(\mathbb{R}^{n}\right)\right.$ ). Therefore, we conclude that $D J_{1}(u)=0$ and $u$ is a weak almost periodic solution of (1.1) by using Proposition 3.7. Hence, the existence is proved.

On the basis of Lemma 3.6, the set of the weak almost periodic solutions of (1.1) is equal to the set $\left\{u \in B^{1,2}\left(\mathbb{R}^{n}\right): D J_{1}(u)=0\right\}$. Since $J_{1}$ is convex, this set is also equal to $\{u \in$ $\left.B^{1,2}\left(\mathbb{R}^{n}\right): J_{1}(u)=\inf J_{1}\left(B^{1,2}\left(\mathbb{R}^{n}\right)\right)\right\}$ which is a convex set. Thus, the set of the weak almost periodic solutions of (1.1) is convex. The proof is complete.

Theorem 5.2 Assume that $\left(\mathrm{H}_{1}\right)-\left(\mathrm{H}_{4}\right)$ hold. Then, for each $e \in A P^{0}\left(\mathbb{R}^{n}\right)$ and for each $\epsilon>0$, there exist an $e_{\epsilon} \in A P^{0}\left(\mathbb{R}^{n}\right)$ and $a u_{\epsilon} \in A P^{2}\left(\mathbb{R}^{n}\right)$ such that $\left\|e-e_{\epsilon}\right\|_{2}<\epsilon$ and

$$
u_{\epsilon}^{\prime \prime}(t)=D_{1} f\left(u_{\epsilon}(t), u_{\epsilon}(-t)\right)+D_{2} f\left(u_{\epsilon}(-t), u_{\epsilon}(t)\right)+e_{\epsilon}(t) .
$$

Proof Consider the operator $\Gamma: B^{2,2}\left(\mathbb{R}^{n}\right) \rightarrow B^{2}\left(\mathbb{R}^{n}\right)$ defined by

$$
\Gamma(u):=\nabla^{2} u-D_{1} f(u(t), u(-t))-D_{2} f(u(-t), u(t)) .
$$

Under $\left(\mathrm{H}_{1}\right)$ and $\left(\mathrm{H}_{2}\right)$, the operators

$$
\left\{\begin{array}{l}
\Gamma_{1}: B^{2,2}\left(\mathbb{R}^{n}\right) \rightarrow B^{2}\left(\mathbb{R}^{n}\right), \\
\Gamma_{1}(u)(t):=D_{1} f(u(t), u(-t)),
\end{array}\right.
$$


and

$$
\left\{\begin{array}{l}
\Gamma_{2}: B^{2,2}\left(\mathbb{R}^{n}\right) \rightarrow B^{2}\left(\mathbb{R}^{n}\right), \\
\Gamma_{2}(u)(t):=D_{2} f(u(-t), u(t)),
\end{array}\right.
$$

are continuous (cf. [7, Theorem 1]). Since the operator $\nabla^{2}: B^{2,2}\left(\mathbb{R}^{n}\right) \rightarrow B^{2}\left(\mathbb{R}^{n}\right)$ is continuous, $\Gamma$ is continuous.

From Theorem 5.1, we know that $\Gamma\left(B^{2,2}\left(\mathbb{R}^{n}\right)\right)=B^{2}\left(\mathbb{R}^{n}\right)$, and so $A P^{0}\left(\mathbb{R}^{n}\right) \subset \Gamma\left(B^{2,2}\left(\mathbb{R}^{n}\right)\right)$. Let $e \in A P^{0}\left(\mathbb{R}^{n}\right)$. Then $e \in \Gamma\left(B^{2,2}\left(\mathbb{R}^{n}\right)\right)$, and thus there exists a $u \in B^{2,2}\left(\mathbb{R}^{n}\right)$ such that $\Gamma(u)=e$. Since $A P^{2}\left(\mathbb{R}^{n}\right)$ is dense in $B^{2,2}\left(\mathbb{R}^{n}\right)$, for each $\epsilon \in(0, \infty)$, there exists a $u_{\epsilon} \in A P^{2}\left(\mathbb{R}^{n}\right)$ such that $\left\|u_{\epsilon}-u\right\|_{2,2}<\epsilon$. An application of continuity of $\Gamma$ implies that $\left\|\Gamma\left(u_{\epsilon}\right)-e\right\|_{2}<\epsilon$. Taking into account that $\Gamma\left(u_{\epsilon}\right) \in A P^{0}\left(\mathbb{R}^{n}\right)$, let $e_{\epsilon}:=\Gamma\left(u_{\epsilon}\right)$. Then $e_{\epsilon}$ and $u_{\epsilon}$ satisfy the desired results. This completes the proof.

\section{Acknowledgements}

The authors express their sincere gratitude to the editors for the careful reading of the original manuscript and useful comments that helped to improve the presentation of the results and accentuate important details.

\section{Funding}

This research is supported by NNSF of P.R. China (Grant Nos. 11771115, 11271106, and 61503171), CPSF (Grant No. 2015M582091), NSF of Shandong Province (Grant No. ZR2016JL021), KRDP of Shandong Province (Grant No. 2017CXGC0701), DSRF of Linyi University (Grant No. LYDX2015BS001), and the AMEP of Linyi University, P.R. China.

\section{Availability of data and materials}

Data sharing not applicable to this article as no datasets were generated or analyzed during the current study.

\section{Competing interests}

The authors declare that they have no competing interests.

\section{Authors' contributions}

All five authors contributed equally to this work. They all read and approved the final version of the manuscript.

\section{Author details}

${ }^{1}$ College of Mathematics and Information Science, Hebei University, Baoding, P.R. China. ${ }^{2}$ Département de Mathématiques, Faculté des Sciences de Gabès, Université de Gabès, Gabès, Tunisia. ${ }^{3}$ School of Basic Sciences, Indian Institute of Technology Mandi, Mandi, India. ${ }^{4}$ Department of Mathematics, University of Peshawar, Peshawar, Pakistan. ${ }^{5}$ LinDa Institute of Shandong Provincial Key Laboratory of Network Based Intelligent Computing, Linyi University, Linyi, P.R. China. ${ }^{6}$ School of Information Science and Engineering, Linyi University, Linyi, P.R. China.

\section{Publisher's Note}

Springer Nature remains neutral with regard to jurisdictional claims in published maps and institutional affiliations.

Received: 14 October 2018 Accepted: 17 December 2018 Published online: 07 January 2019

\section{References}

1. Aftabizadeh, A.R., Huang, Y.K., Wiener, J.: Bounded solutions for differential equations with reflection of the argument. J. Math. Anal. Appl. 135, 31-37 (1988)

2. Alexeev, V.M., Tihomirov, V.M., Fomin, S.V.: Commande Optimale, French edn. MIR, Moscow (1982)

3. Ayachi, M., Lassoued, D.: On the existence of Besicovitch almost periodic solutions for a class of neutral delay differential equations. Facta Univ., Ser. Math. Inform. 29, 131-144 (2014)

4. Besicovitch, A.S.: Almost Periodic Functions. Cambridge University Press, Cambridge (1932)

5. Blot, J.: Calculus of variations in mean and convex Lagrangians. J. Math. Anal. Appl. 134, 312-321 (1988)

6. Blot, J.: Une approche variationnelle des orbites quasi-périodiques des systèmes hamiltoniens. Ann. Sci. Math. Qué. 13, 7-32 (1990) (French)

7. Blot, J.: Oscillations presque-périodiques forcées d'équations d'Euler-Lagrange. Bull. Soc. Math. Fr. 122, 285-304 (1994) (French)

8. Blot, J., Lassoued, D.: Bumps of potentials and almost periodic oscillations. Afr. Diaspora J. Math. 12, 122-133 (2011)

9. Bohr, H.: Almost Periodic Functions. Chelsea, New York (1956)

10. Brézis, H.: Analyse Fonctionnelle. Théorie et Applications. Masson, Paris (1983) (French)

11. Busse, C., Lassoued, D., Nguyen, T.L., Saierli, O.: Exponential stability and uniform boundedness of solutions for nonautonomous periodic abstract Cauchy problems. An evolution semigroup approach. Integral Equ. Oper. Theory 74, 345-362 (2012) 
12. Corduneanu, C.: Almost Periodic Functions, 2nd English edn. Chelsea, New York (1989)

13. de Figueiredo, D.G.: The Ekeland Variational Principle with Applications and Detours, Tata Institute of Fundamental Research, Bombay. Springer, Berlin (1989)

14. Fink, A.M.: Almost Periodic Differential Equations. Lecture Notes in Mathematics. Springer, Berlin (1974)

15. Gupta, C.P.: Boundary value problems for differential equations in Hilbert spaces involving reflection of the argument. J. Math. Anal. Appl. 128, 375-388 (1987)

16. Gupta, C.P.: Existence and uniqueness theorems for boundary value problems involving reflection of the argument. Nonlinear Anal. 11, 1075-1083 (1987)

17. Gupta, C.P.: Two-point boundary value problems involving reflection of the argument. Int. J. Math. Math. Sci. 10, 361-371 (1987)

18. Hai, D.D.: Two point boundary value problem for differential equations with reflection of argument. J. Math. Anal. Appl. 144, 313-321 (1989)

19. Lassoued, D.: Exponential dichotomy of nonautonomous periodic systems in terms of the boundedness of certain periodic Cauchy problems. Electron. J. Differ. Equ. 2013, 89 (2013)

20. Lassoued, D.: New aspects of nonautonomous discrete systems stability. Appl. Math. Inf. Sci. 9, 1693-1698 (2015)

21. Levitan, B.M., Zhikov, V.V.: Almost Periodic Functions and Differential Equations. Cambridge University Press, Cambridge (1982)

22. O'Regan, D.: Existence results for differential equations with reflection of the argument. J. Aust. Math. Soc. A 57, 237-260 (1994)

23. Piao, D.: Periodic and almost periodic solutions for differential equations with reflection of the argument. Nonlinear Anal. 57, 633-637 (2004)

24. Piao, D.: Pseudo almost periodic solutions for differential equations involving reflection of the argument. J. Korean Math. Soc. 41, 747-754 (2004)

25. Piao, D., Sun, J.: Besicovitch almost periodic solutions for a class of second order differential equations involving reflection of the argument. Electron. J. Qual. Theory Differ. Equ. 2014, 41 (2014)

26. Šarkovskii, A.N.: Functional-differential equations with a finite group of argument transformations. In: Asymptotic Behavior of Solutions of Functional-Differential Equations, pp. 118-142, 157. Akad. Nauk Ukrain. SSR, Inst. Mat., Kiev (1978)

27. Vo-Khac, K.: Étude des fonctions quasi-stationnaires et de leurs applications aux équations différentielles opérationnelles. Bull. Soc. Math. France Mém. 6 (1966) (French)

28. Wang, Y., Zada, A., Ahmad, N., Lassoued, D., Li, T.: Uniform exponential stability of discrete evolution families on space of p-periodic sequences. Abstr. Appl. Anal. 2014, Article ID 784289 (2014)

29. Wiener, J., Aftabizadeh, A.R.: Boundary value problems for differential equations with reflection of the argument. Int. J. Math. Math. Sci. 8, 151-163 (1985)

30. Zima, M.: On positive solutions of functional-differential equations in Banach spaces. J. Inequal. Appl. 6, 359-371 (2001)

\section{Submit your manuscript to a SpringerOpen ${ }^{\circ}$ journal and benefit from:}

- Convenient online submission

- Rigorous peer review

- Open access: articles freely available online

- High visibility within the field

- Retaining the copyright to your article

Submit your next manuscript at $>$ springeropen.com 